\title{
Does Linearity Always Hold Between Friction Force and Real Contact Area: An Experimental Study
}

\author{
X. M. Liang, Y. Z. Xing, L. T. Li, W. K. Yuan and G. F. Wang* \\ Department of Engineering Mechanics, SVL and MMML, Xi'an Jiaotong University, \\ Xi'an 710049, China \\ *E-mail: wanggf@mail.xjtu.edu.cn
}

\begin{abstract}
Classical laws of friction suggest that friction force is proportional to the normal load and independent of the nominal contact area. As a great improvement in this subject, it is now widely accepted that friction force is proportional to the real area in contact, and much work has been conducted based on this hypothesis. In present study, this hypothesis will be carefully revisited by measuring the friction force and real contact area in-site and real-time at both normal loading and unloading stages. Our experiments reveal that the linear relation always holds between friction force and normal load. However, for the relation between friction force and real contact area, the linearity holds only at the loading stage while fails at the unloading stage. This study may improve our understanding of the origin of friction.
\end{abstract}

Key words: Friction Force, Real Contact Area, Rough Surface 


\section{Introduction}

Approximate 20 percent of the global energy consumption is attributed to the harmful friction [1], and it is of practical significance to improve the fundamental understanding of friction. Back to 1493, Leonardo da Vinci observed that the friction force between two solids is proportional to the applied normal load and independent of the nominal contact area [2]. The proportionality was hereby defined as the friction coefficient. As a great advance in the modern understanding of friction, Bowden and Tabor [3] argued that the tangential force required to slide each contact junction is proportional to the junction area by a critical shear strength, which leads to the linearity between friction force and the real contact area. This concept could explain the origin of the ancient phenomenological friction law if the linearity between real contact area and normal load is fulfilled.

After the work of Bowden and Tabor, study on the contact of rough surfaces has experienced a shriving development for determining the relation between real contact area and normal load. There are multi-asperities models [4-6], fractal models [7-9], Persson theory [10, 11], etc. Interestingly, almost all these theories showed approximately linear load-area relationship within a certain contact fraction [12]. Such linearity was also verified through finite element analysis [13-15] and atomic simulations $[16,17]$.

Besides, huge efforts have been devoted into experimental measurements of the real contact area under a given pressure [18]. Indirect methods such as measuring the thermal resistance [19], electric resistance [20] and ultrasonic reflection [21] were adopted to evaluate the contact fraction. To obtain a rather detailed picture of contact spots, techniques as X-ray computed tomography [22], optical interference [23-25], fluorescent molecule probes [26], and frustrated total internal reflection [27-29] have been used for recording the real contact area. Likewise, almost all experiments [22, 24, 27-29] reported the linear dependence of real contact area on normal load.

In view of the linear load-area relationship in the continuous loading process, it seems settled that linearity exists between any two of friction force, real contact area, and normal load. Nonetheless, such linearity of load-area relation might break down for some circumstances. For the contact between viscoelastic rubber sphere and glass, a strong hysteresis was found in the load-area curves during the loading-unloading circle [24]. A similar hysteresis was observed for the contact of rough copper sample against smooth glass on account of the plastic deformation [29]. During the unloading process, the real contact area decreases monotonously but nonlinearly with respect to load, which may provide a scenario to test whether friction force exclusively depends linearly on contact area or load.

In this study, the friction tests are conducted between copper samples and a glass disc. The frustrated total internal reflection method is adopted to measure the real contact area under various external normal load and friction force. The results verify the linear dependence of friction force on both normal load and real contact area during the normal loading stage. Nonetheless, for the unloading stage, such linearity still holds between friction force and normal load, whereas a nonlinear relation between friction force and contact area is observed.

\section{Experiment}

\subsection{Sample Preparation}

Three cubic samples made of copper were prepared for the contact and friction tests. As shown in Fig. 1, the surfaces to be tested were first rubbed with sandpaper to obtain rough topographies. Such surface topographies are quite common for friction 
pairs. The nominal contact region of each sample surface is of $3 \times 3 \mathrm{~mm}^{2}$. Prior to the contact-friction tests, standard tensile test was carried out, by which the Young's modulus $E$, the Poisson's ratio $v$, and the yield stress $\sigma_{y}$ were measured as $125.4 \mathrm{GPa}$, 0.34 , and 225.3 $\mathrm{MPa}$, respectively.
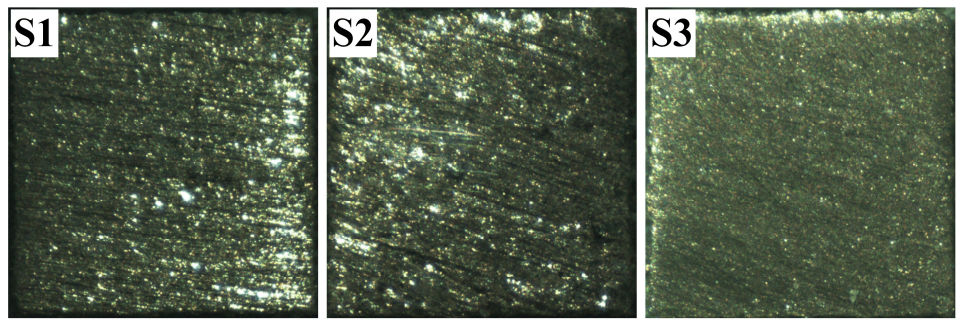

Figure 1 Surfaces of test samples S1, S2, and S3

\subsection{Experimental Apparatus}

As schematically shown in Fig. 2, a new apparatus was designed and built to measure friction force, real contact area and normal load simultaneously. The apparatus consists of three basic parts: the loading system, the illuminating system, and the camera shooting system.

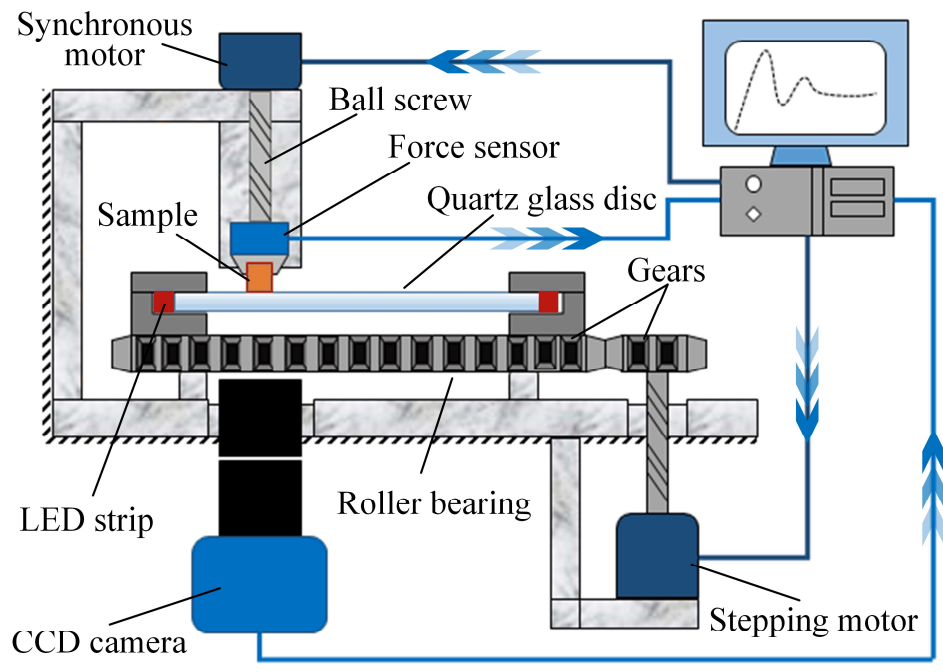

Figure 2 Schematic diagram of the experimental apparatus

The main module of the loading system is a roller bearing with its inner-ring screwed on a work bench and outer-ring installed with a quartz glass disc. The quartz glass disc is $200 \mathrm{~mm}$ in diameter and $15 \mathrm{~mm}$ in thickness. A stepping motor is connected with the outer-ring of the bearing through a pair of gears, and the glass disc can rotate steadily with the torque transmitted. The test sample is fixed on a sample holder, which is motivated by a ball screw and a synchronous motor. During contact, the sample will be compressed against the upper surface of the quartz glass at about $40 \mathrm{~mm}$ offset from the disc center. When the stepping motor is triggered, the sample will be rubbed by the glass disc, and both shear force and normal load are recorded by a three-dimensional force sensor connected with the sample holder. The loading rate of the sample holder is $0.5 \mathrm{~mm} / \mathrm{min}$, and the rotating speed of the glass disk is 0.02 $\mathrm{rpm}$. The data acquisition rate of the force sensor is set as $30 \mathrm{~Hz}$.

The illuminating system is constructed based on the frustrated total internal 
refection technique. A monochromatic violet light-emitting diode strip is wrapping around the glass disc circumferentially. All lights incident into the glass disc from the flank. The light strip and the edge of the glass disc are together covered with a blackout fabric. Lights incident at an angle smaller than the critical total reflection angle are absorbed by the fabric, whereas others constrained within the glass disc. The entire apparatus is placed in a dark room to prevent interference from other light source.

The function of the camera shooting system records the evolution of the real contact area. A charge-coupled device (CCD) camera is installed beneath the glass disc and calibrated to focus on the upper surface of the glass disc. Since the glass disc is much harder than the test samples, its upper surface can be considered as rigid flat plane, which is easy to focus on during compressing and rubbing. When test sample is compressed against the glass surface, the total internal reflection condition will be broken at the area of intimate contact, and light will be scattered by the sample surface and subsequently captured by the CCD camera [28, 29]. Within the non-contact region, light will be reflected at an angle larger than the critical total reflection angle and still trapped inside the glass disc. Once activated, the CCD camera will record the images of the contact interface consecutively at a constant rate of $30 \mathrm{fps}$. With a $1 \times$ objective lens installed on the camera, each pixel on the image corresponds to a squared region of $4.8 \times 4.8 \mathrm{um}^{2}$ on the contact interface.

By adopting the improved Otsu technique [30], the original images could be transformed into binary images, with 1 representing for contact spots and 0 for non-contact spots. Hence, the real contact area is obtained as the amount of the contact spots times the area of a single spot. Through triggering both camera and force sensor at the same time, the real contact area, the friction force, and the normal load are measured and recorded synchronously.

\subsection{Experimental Procedure}

Full normal loading-unloading tests were performed on samples S1, S2, and S3. In the loading stages, samples were driven by the synchronous motor and brought into contact against the glass disc, with the normal load $P$ gradually increasing from 0 to $120 \mathrm{~N}$. Then the synchronous motor was reversed, whereupon the normal load $P$ decreased till vanishing. During the loading-unloading circle for each sample, a series of rubbing processes were carried out through rotating the glass disc at specific normal loads of $20 \mathrm{~N}, 40 \mathrm{~N}, 60 \mathrm{~N}, 80 \mathrm{~N}$, and $100 \mathrm{~N}$. In each rubbing process, there is a slight increment of contact area $A_{r}$, which has also been found in Ref. [31]. To avoid the significant increase of contact area incurred by long time of friction, the rotation of glass disc was immediately halted once the friction force converged to a stable value, the kinetic friction force $F_{k}$. Before that, a maximum static friction force $F_{s}$ can be clearly observed in each rubbing process. The normal load $P$, the friction force $F$, and the real contact area $A_{r}$ were continuously measured and recorded in the whole test. For comparison, $P, F_{s}$ and $F_{k}$ are normalized by $E^{*} A_{0}$, and $A_{r}$ normalized by $A_{0}$, where $E^{*}=E /\left(1-v^{2}\right)$ is the composite elastic modulus and $A_{0}$ represents the nominal contact area.

\section{Results and Discussions}

Fig. 3 displays the evolution of the contact fraction $A_{r} / A_{0}$ with respect to the dimensionless normal load $P /\left(E^{*} A_{0}\right)$. There are five small jumps in each curve at the loads conducting rubbing processes, where the real contact area endures a small increment owing to friction. For sample S1 under the load $P=20 \mathrm{~N}$, the contact 
regions at the initiation and the end of the rubbing are shown in Fig. 4(a) and 4(b), respectively. After rubbing, some contact spots have grown larger, along with new ones' coming into being. Basically, $A_{r} / A_{0}$ increases approximately linearly with $P /\left(E^{*} A_{0}\right)$ in the loading stages, and the dimensionless mean pressures $P /\left(E^{*} A_{r}\right)$ for samples S1, S2 and S3 are best fitted with $0.0053,0.0035$ and 0.0023 , respectively. However, as the plastic deformation accumulated in the loading stage cannot be fully recovered, the real contact area decreases monotonously during unloading but nonlinearly till vanishing.

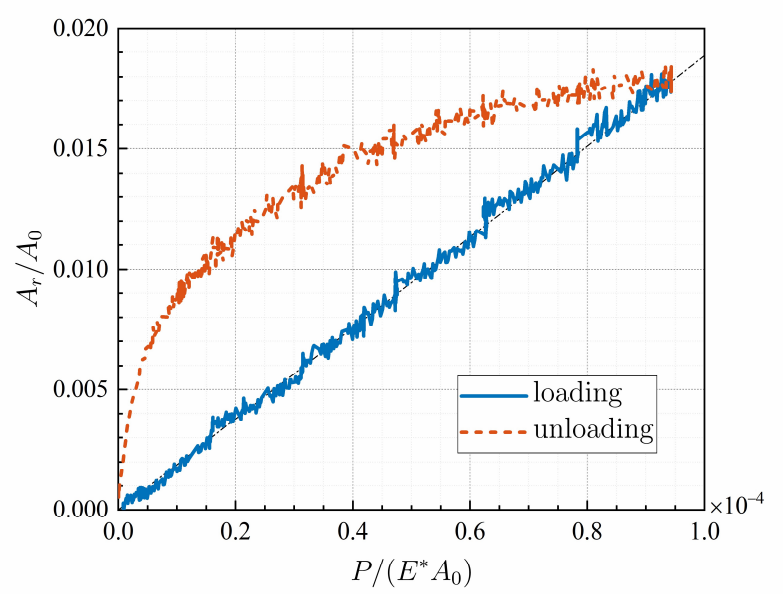

(a)

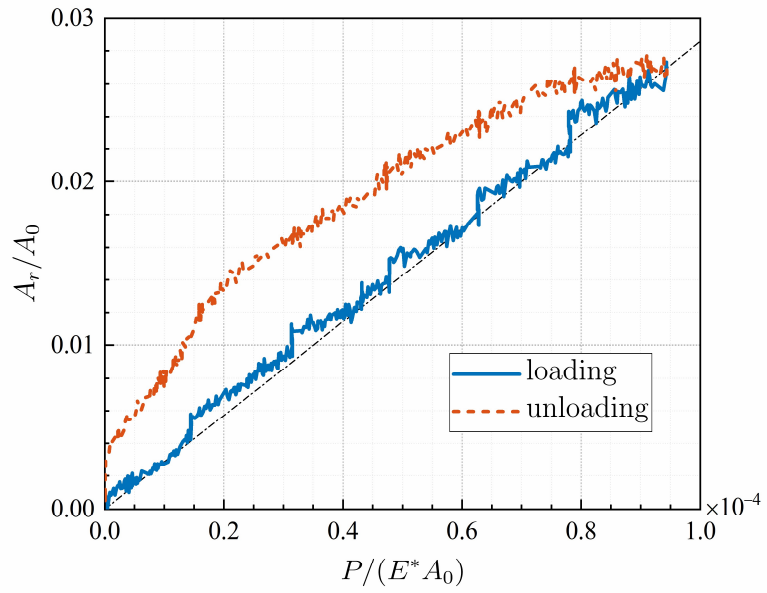

(b) 


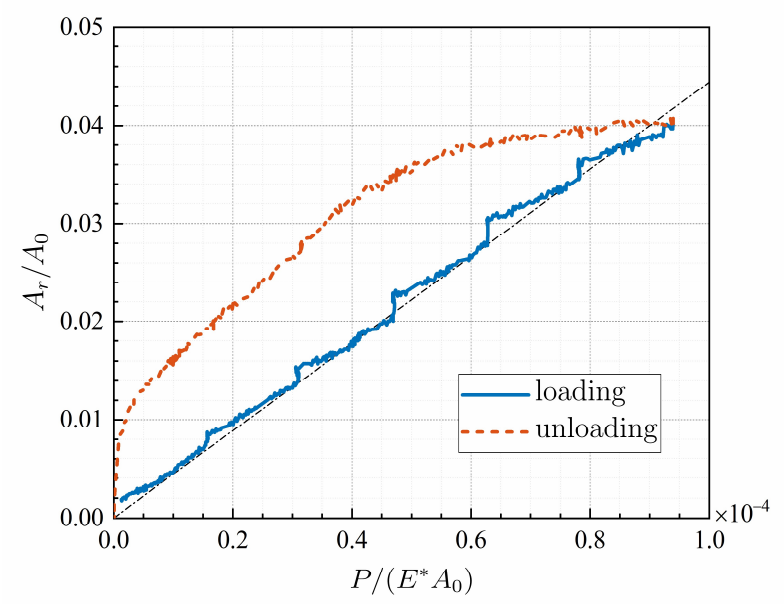

(c)

Figure 3 Evolution of the contact fraction $A_{r} / A_{0}$ with respect to the dimensionless normal load $P /\left(E^{*} A_{0}\right)$ : (a) sample $\mathrm{S} 1$, (b) sample $\mathrm{S} 2$, (c) sample $\mathrm{S} 3$
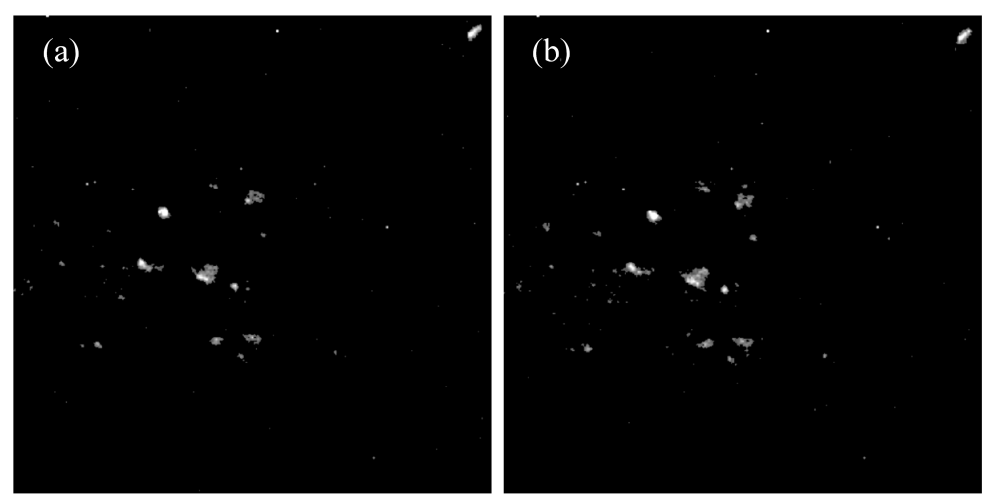

Figure 4 The real contact areas of sample S1 at the: (a) initiation of the first rubbing, (b) end of the first rubbing

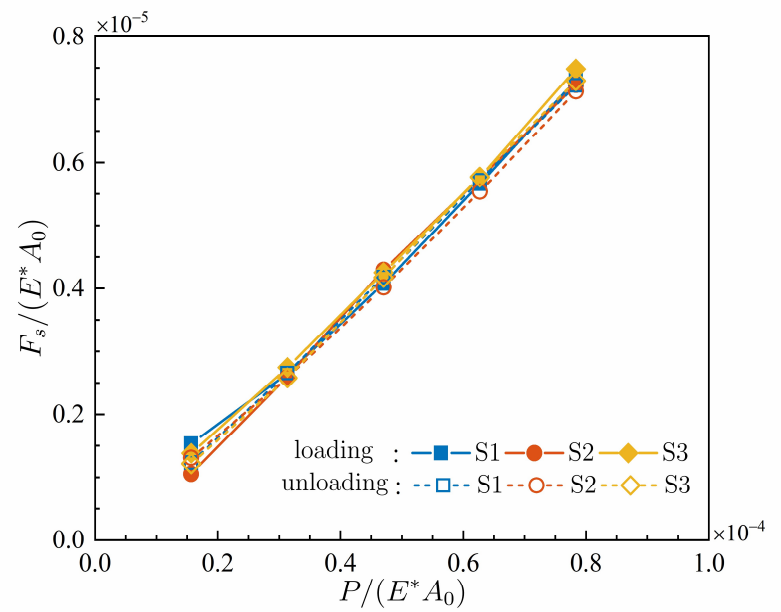

Figure 5 The dimensionless maximum static friction force $F_{s} /\left(E^{*} A_{0}\right)$ at various dimensionless normal loads $P /\left(E^{*} A_{0}\right)$

For various normal loads, the dimensionless maximum static friction force $F_{S} /\left(E^{*} A_{0}\right)$ is presented in Fig. 5. $F_{S} /\left(E^{*} A_{0}\right)$ is clearly enlarged as the normal load 
increases. For any specific normal load during both loading and unloading, $F_{s} /\left(E^{*} A_{0}\right)$ hardly varies among different samples. It appears that both the loading history and rough topography of the samples have little effect on the static friction. Fig. 6 displays the dimensionless kinetic friction force $F_{k} /\left(E^{*} A_{0}\right)$ with respect to the dimensionless normal load $P /\left(E^{*} A_{0}\right)$. The kinetic friction force $F_{k}$ exhibits a similar dependence on normal load as the static friction force $F_{s}$ does, except that $F_{k}$ is a little bit smaller than $F_{s}$ under the same pressure.

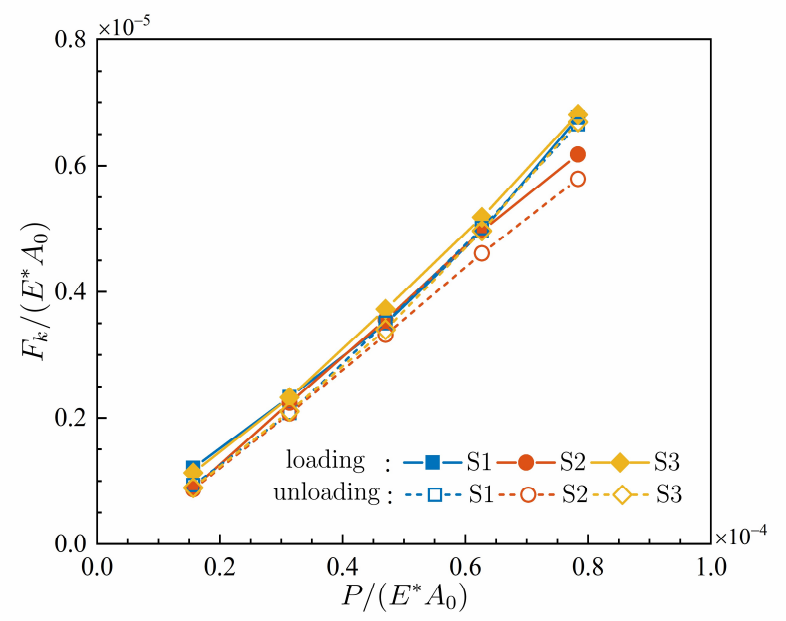

Figure 6 The dimensionless kinetic friction force $F_{k} /\left(E^{*} A_{0}\right)$ at various dimensionless normal loads $P /\left(E^{*} A_{0}\right)$

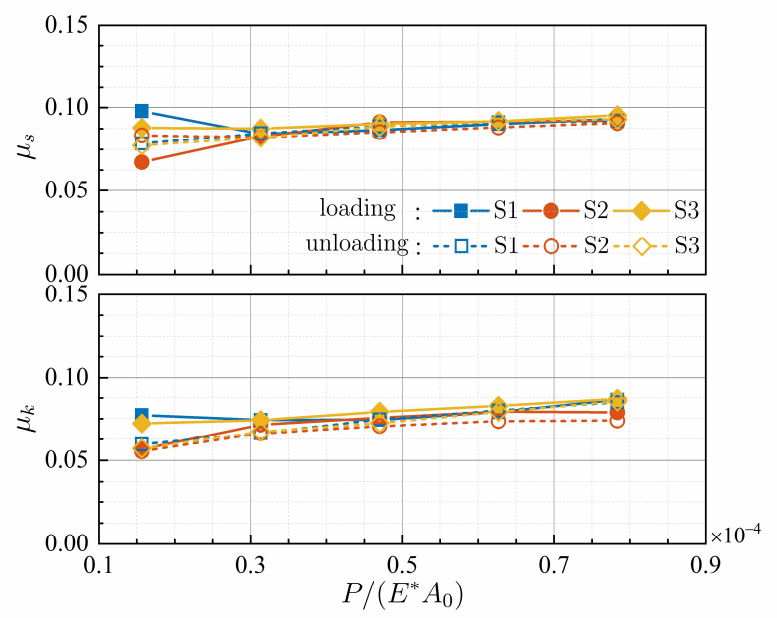

Figure 7 Evolution of the friction coefficient with respect to the dimensionless normal load

For a clearer insight into the relation between the friction force and the external normal load, the static friction coefficient $\mu_{s}$ and the kinetic friction coefficient $\mu_{k}$ are calculated, as shown in Fig. 7. It is seen that, in both loading and unloading process, $\mu_{s}$ and $\mu_{k}$ keep almost constant, which proves the proportionality between friction force and normal load. 


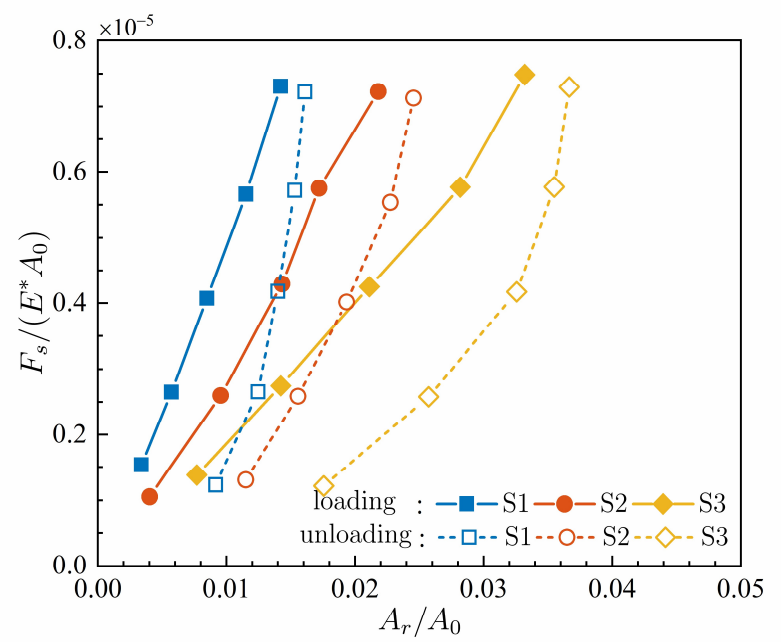

Figure 8 The dimensionless maximum static friction force $F_{s} /\left(E^{*} A_{0}\right)$ under various contact fractions $A_{r} / A_{0}$

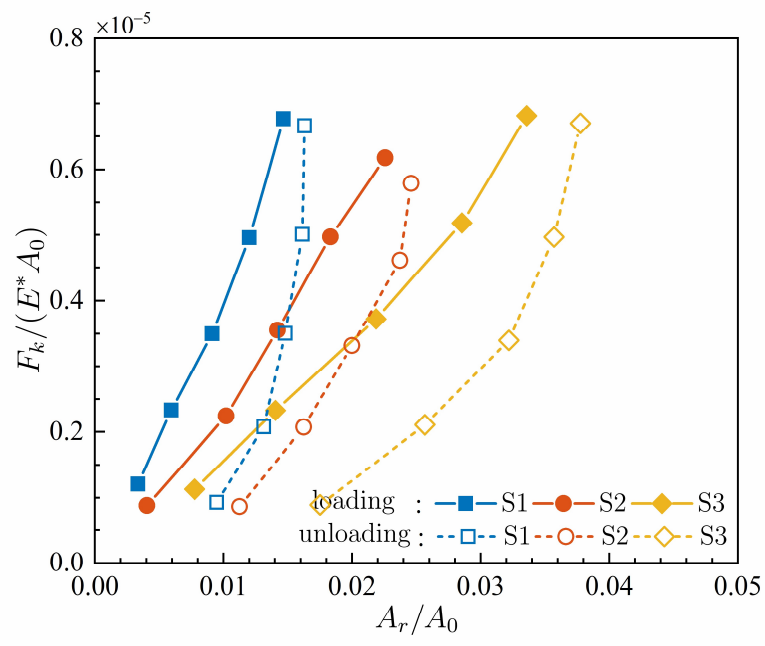

Figure 9 The dimensionless kinetic friction force $F_{k} /\left(E^{*} A_{0}\right)$ under various contact fractions $A_{r} / A_{0}$

The evolutions of $F_{s} /\left(E^{*} A_{0}\right)$ and $F_{k} /\left(E^{*} A_{0}\right)$ versus the contact fraction $A_{r} / A_{0}$ are shown in Figs. 8 and 9, respectively. Both friction forces vary in a quite similar pattern with the evolution of the contact fraction. At five specific loads in the loading stage, the frictional force is basically proportional to the contact area. Such linearity was also observed at the onset of friction between PDMS and glass plate [32]. Nevertheless, under the same normal load, thus almost the same frictional force, it is observed that the contact area in the unloading stage is much larger than that in the loading stage. 


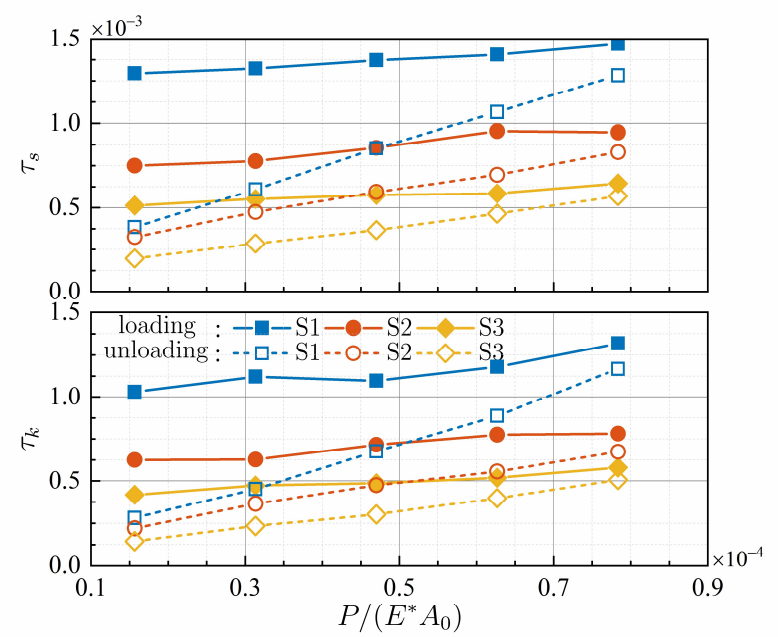

Figure 10 Evolution of the dimensionless mean tangential stress with respect to the dimensionless normal load

For both static friction and kinetic friction, the dimensionless mean tangential stresses $F_{s} /\left(G A_{r}\right)$ and $F_{k} /\left(G A_{r}\right)$ are calculated and denoted as $\tau_{s}$ and $\tau_{k}$, respectively, with $G=E /[2(1+v)]$ being the shear modulus. Fig. 10 displays $\tau_{s}$ and $\tau_{k}$ at various normal loads for both loading and unloading. It can be seen that both $\tau_{s}$ and $\tau_{k}$ change slightly during loading, while decrease monotonously in the unloading stages. The almost constant mean tangential stress at loading stages suggests that the friction force is approximately proportional to the real contact area. It is also noticed that $\tau_{s}$ and $\tau_{k}$ vary significantly among different samples, which indicates that even for loading the proportional coefficient between friction force and the real contact area is strongly dependent on the rough topographies. A probable explanation is that the variation of the rough topographies results in the variation of the mean pressure on the real contact area, and the mean tangential stress is proportional to the mean pressure by the friction coefficient. Either way, linear dependency does not always hold between friction force and the real contact area, given the fact that the same friction force corresponds to two different values of real contact area during loading and unloading.

\section{Conclusions}

By adopting the frustrated total internal reflection method, an apparatus has been built to measure the friction force, the real contact area, and the external normal load in-situ and real-time. Loading-unloading tests are conducted on various copper samples. At several specified normal loads, samples are rubbed for a short period of time in both loading and unloading stages. The experiment results suggest that the friction force is roughly proportional to both the normal load and the real contact area during loading. Such linearity still holds between friction force and normal load during unloading, while fails for the relation between friction force and real contact area. These results illuminate that the linear assumption of friction force on contact area does not always hold.

\section{Acknowledgements}

Supports from the National Natural Science Foundation of China (Grant No. 11525209) are acknowledged. 


\section{References}

[1] K. Holmberg, A. Erdemir, Influence of tribology on global energy consumption, costs and emissions, Friction 5 (3) (2017) 263-284.

[2] I.M. Hutchings, Leonardo da Vinci's studies of friction, Wear 360-361 (2016) 51-66.

[3] F.P. Bowden, D. Tabor, The area of contact between stationary and between moving surfaces, Proc. R. Soc. A-Math. Phys. Eng. Sci. 169 (938) (1939) 391-413.

[4] J.F. Archard, Elastic deformation and the law of friction, Proc. R. Soc. A-Math. Phys. Eng. Sci. 243 (1233) (1957) 190-205.

[5] J.A. Greenwood, J.B.P. Williamson, Contact of nominally flat surfaces, Proc. R. Soc. A-Math. Phys. Eng. Sci. 295 (1442) (1966) 300-319.

[6] A.W. Bush, R.D. Gibson, T.R. Thomas, The elastic contact of a rough surface, Wear 35 (1) (1975) 87-111.

[7] A. Majumdar, B. Bhushan, Fractal model of elastic-plastic contact between rough surfaces, J. Tribol.-Trans. ASME 113 (1) (1991) 1-11.

[8] M. Ciavarella, G. Demelio, J.R. Barber, Y.H. Jang, Linear elastic contact of the Weierstrass profile, Proc. R. Soc. A-Math. Phys. Eng. Sci. 456 (1994) (2000) 387-405.

[9] R.L. Jackson, J.L. Streator, A multi-scale model for contact between rough surfaces, Wear 261 (11) (2006) 1337-1347.

[10]B.N.J. Persson, Theory of rubber friction and contact mechanics, J. Chem. Phys. 115 (8) (2001) 3840-3861.

[11]B.N.J. Persson, Elastoplastic contact between randomly rough surfaces, Phys. Rev. Lett. 87 (11) (2001) 116101.

[12] G. Carbone, F. Bottiglione, Asperity contact theories: do they predict linearity between contact area and load? J. Mech. Phys. Solids 56 (8) (2008) 2555-2572.

[13] S. Hyun, L. Pei, J.F. Molinari, M.O. Robbins, Finite-element analysis of contact between elastic self-affine surfaces, Phys. Rev. E 70 (2) (2004) 026117.

[14]L. Pei, S. Hyun, J.F. Molinari, M.O. Robbins, Finite element modeling of elasto-plastic contact between rough surfaces, J. Mech. Phys. Solids 53 (11) (2005) 2385-2409.

[15]H. Song, E. Van der Giessen, X. Liu, Strain gradient plasticity analysis of elasto-plastic contact between rough surfaces, J. Mech. Phys. Solids 96 (2016) 18-28.

[16]B.Q. Luan, M.O. Robbins, Hybrid atomistic/continuum study of contact and friction between rough solids, Tribol. Lett. 36 (1) (2009) 1-16.

[17]S. Solhjoo, A.I. Vakis, Continuum mechanics at the atomic scale: insights into non-adhesive contacts using molecular dynamics simulations, J. Appl. Phys. 120 (21) (2016) 215102.

[18]K.L. Woo, T.R. Thomas, Contact of rough surfaces: a review of experimental work, Wear 58 (2) (1980) 331-340.

[19]M.R. Sridhar, M.M. Yovanovich, Review of elastic and plastic contact conductance models: comparison with experiment, J. Thermophys. Heat Transf. 8 (4) (1994) 633-640.

[20]B. Bhushan, The real area of contact in polymeric magnetic media-II: experimental data and analysis, ASLE Trans. 28 (2) (1985) 181-197.

[21]R.S. Dwyer-Joyce, B.W. Drinkwater, A.M. Quinn, The use of ultrasound in the investigation of rough surface interfaces, J. Tribol.-Trans. ASME 123 (1) (2001) 8-16. 
[22]F.K. Zhang, J.H. Liu, X.Y. Ding, R.L. Wang, Experimental and finite element analyses of contact behaviors between non-transparent rough surfaces, J. Mech. Phys. Solids 126 (2019) 87-100.

[23]A. Ovcharenko, G. Halperin, I. Etsion, M. Varenberg, A novel test rig for in situ and real time optical measurement of the contact area evolution during pre-sliding of a spherical contact, Tribol. Lett. 23 (1) (2006) 55-63.

[24]B.A. Krick, J.R. Vail, B.N.J. Persson, W.G. Sawyer, Optical in situ micro tribometer for analysis of real contact area for contact mechanics, adhesion, and sliding experiments, Tribol. Lett. 45 (1) (2012) 185-194.

[25]B.B. Zugelj, M. Kalin, In-situ observations of a multi-asperity real contact area on a submicron scale, Strojniški Vestn.-J. Mech. Eng. 63 (6) (2017) 351-362.

[26]B. Weber, T. Suhina, T. Junge, L. Pastewka, A.M. Brouwer, D. Bonn, Molecular probes reveal deviations from Amontons' law in multi-asperity frictional contacts, Nat. Commun. 9 (1) (2019) 808.

[27] S.M. Rubinstein, G. Cohen, J. Fineberg, Detachment fronts and the onset of dynamic friction, Nature 430 (7003) (2004) 1005-1009.

[28] A.I. Bennett, K.L. Harris, K.D. Schulze, J.M. Uruena, A.J. McGhee, A.A. Pitenis, M.H. Müser, T.E. Angelini, W.G. Sawyer, Contact measurements of randomly rough surfaces, Tribol. Lett. 65 (4) (2017) 134.

[29] L.T. Li, X.M. Liang, Y.Z. Xing, D. Yan, G.F. Wang, Measurement of real contact area for rough metal surfaces and the distinction of contribution from elasticity and plasticity, J. Tribol.-Trans. ASME 143 (7) (2021) 071501.

[30]B.J. Song, S.Z. Yan, W.W.K. Xiang, A measurement method for distinguishing the real contact area of rough surfaces of transparent solids using improved Otsu technique, Chin. Phys. B 24 (1) (2015) 014601.

[31]I. Etsion, Revisiting the Cattaneo-Mindlin concept of interfacial slip in tangentially loaded compliant bodies, J. Tribol.-Trans. ASME 132 (2) (2010) 020801.

[32]R. Sahli, G. Pallares, C. Ducottet, I.E. Ben Ali, S. Al Akhrass, M. Guibert, J. Scheibert, Evolution of real contact area under shear and the value of static friction of soft materials, Proc. Natl. Acad. Sci. U. S. A. 115 (3) (2018) 471-476. 